\title{
Optical properties of (ZnTiO3)/Epoxy Nanocomposite Thin films
}

\author{
Warqaa A. Shakir ${ }^{1}$, Sawsan H. Abdullah ${ }^{2}$, Falah I. Mustafa ${ }^{3}$, Khalid Kh.Ateia ${ }^{4}$, Amnah M.Ibraheem ${ }^{5}$
}

Solar energy research center, Renewable energy Directorate, Higher education scientific research and Science Technology Ministry, Baghdad, Iraq ${ }^{1,2,3,4,5}$

\begin{abstract}
Two layers of epoxy reinforced with nano- Zinc Tetanet (ZnTiO3) uniform thin films have been prepared using a spin coating technique on glass floors, dispersion and magnetic stirring techniques are used to prepare the nanocomposites, FTIR was employed to aid interpretation of results with a fixed thickness and proportions by weight $(0 \mathrm{Wt} \%, 1 \mathrm{Wt} \%, 2 \mathrm{Wt} \%, 3 \mathrm{Wt} \%, 4 \mathrm{Wt} \%) \mathrm{ZnTiO} 3 a d d i t i v e s$. The optical characteristics were studied by measuring the transmittance and absorbance spectrometry as a function of the wavelength (200-1100) nanometers. The results of the transmittance were very high, and high transparency in infrared (IR) radiation was measured by the FTIR and UV-VIS measurements. The optical properties included the calculations of the energy gap; some optical constants were also carried out, which included absorption coefficient making it Suitable for applications of solar cells
\end{abstract}

Keywords: Nanocomposites, Epoxy, nanoZnTiO3, thin film, Spin coating, optical properties.

\section{Introduction}

The main reason beyond the out spread use of polymers is their unique set of properties such as stiffness, light weight, low cost, and ease of processing and fabrication which med it one of the important categories of materials finding their way into the electronic industries [1]. The long-term goal of this research is to realize the potential that organic photovoltaic's (OPV) have as a low-cost alternative to conventional photovoltaic's and others have a power conversion efficiency of 5\% [2] nanocomposite is a special composite where one of the phases has one, two or three dimensions less than 100nm [3]. An important parameter for characterizing the effectiveness of reinforcement is the ratio of surface to area (A) of reinforcement to volume of reinforcement (V) [4]. $\mathrm{ZnO}$ and $\mathrm{TiO} 2$ are the most important nano filler Oxides used in (PV technology) it is used as a transparent conductive oxide (TCOs).Zinc oxide is a direct gap semiconductor $3.4 \mathrm{ev}$, with a considerable fraction of ionic bonding [5] $\mathrm{TiO}_{2}$ is a semiconductor of type $\mathrm{n}$ and has a forbidden band ranging between $3.46 \mathrm{ev}$ and $3.04 \mathrm{ev}$ and has high quality resistance At $25^{\circ} \mathrm{C}$, it has high transparency in the visible field and a good reflection of the infrared radiation [6, 7]. (Mullerov'a, (2017) studies of a (optical properties of zinc titanate ZnTiO3prepared by reactive RF sputtering [8], (H. Hafez,(2005) determinate Highly Efficient Dye-sensitized Solar Cells Based on Single Crystalline TiO2 Nanorod Film [9].Our goal of this work is to develop materials and devices that are capable of making large Improvements in efficiency of polymer solar cells

\section{Theoretical Considerations}

Optical properties of the nano system (ZnTiO3) Thin-films models will be measured absorption coefficient determined by using equation (2) Energy gap, electron transition also determined. The absorption coefficient $(\alpha)$ is defined as the ratio of the decrease in the energy of the radiation to the unit of distance toward the propagation of the wave within the medium, It depends on the energy of the falling photons, the wave length, the nature of the membrane surface, the energy gap of the semiconductor and the type of electronic transitions that occur between energy bands [10] Light intensity (I) is given at thickness (t) from the surface of the semiconductor using the following formula [11]

$\mathrm{I}=\mathrm{Io} \exp (-\alpha \mathrm{t})$ 
The following equation was used to calculate the absorption coefficient of the membrane used in the research $[12,13]$

$\alpha=2.303 \mathrm{~A} / \mathrm{t}$

Whereas:

\section{A: Absorption $\left(\mathrm{cm}^{-1}\right)$}

The absorption coefficient of the membrane material $: \alpha$

t: Thin film thickness

The optical energy gap was calculated for the indirect allowed transmission by applying relationship (3) by drawing the relationship between $(\alpha h v)^{2}$ as a function of photon energy and (hv) [14]. The intersection of the straight segment of the curve with the photon energy axis represents the value of the optical energy gap[15].

$\alpha h v=A(h v-E g)^{r}$

Whereas:

: Absorption coefficient, $\quad(v)$ : frequency of falling photon $) \alpha($

, A: constant, (h) Blanck constant

Eg: energy gap

By simplifying the equation for in direct transmission we get

$\left.(4)^{r}\right) g(\alpha h v)^{2}=A^{2}(h v-E$

r: The exponential coefficient depends on the type of transition

If $r$ : equal 1/2 allowed Direct transmission or $3 / 2$ forbidden Direct transmission

Either if they $\mathrm{r}=2$ allowed indirect transmission or $\mathrm{r}=3$ forbidden indirect transmission

Where $r$ here equal $3 / 2$

Annealing: It is the process of exposing the thin film to a certain temperature and for a period of time.

Thickness was calculated using the gravimetric method from the following equation

$\mathrm{m} 1-\mathrm{m} 2 / \rho$. A $(5) t=$

$\mathrm{t}$ : thickness of the thin film

m1-m2: difference in weight before and after deposition

$\rho$ : density of polymer nanocomposite

A: surface area of substrate

\section{Experimental Work 3.}

Epoxy resin(105) It is a liquid For processing from the liquid to solid state by adding hardener (3:2 wt $\%)$ used as a (matrix material) for the preparation of nano composite material, nanocomposites are prepared by dispersing nano powder (reinforced material) particle size of $(\mathrm{ZnTiO} 3)$ is $(50-80) \mathrm{nm}$, it is a white powder that does not dissolve in water purity, $(99 \%)$ and proportions by weight $(0 \mathrm{Wt} \%, 1 \mathrm{Wt} \%, 2 \mathrm{Wt} \%, 3 \mathrm{Wt} \%)$ to get (nanocomposite material) spin coating technique using to achieve better state of dispersion, first the nano particles were treated with alcoholic medium (ethanol or acetone) for the deagglomeration of the nanoparticle bundles. The treated nanoparticles are then added to the Epoxy resin followed by magnetic stirring (100 rpm) for an hour to homogenization followed by spin coating (1500) rpm for (30 sec) time., one type of glass is used of dimensions $(2.5 * 2.5) \mathrm{cm} 2$ (German origin). After the process of selecting the models of glass bases wash thoroughly with water, then washed with hydrolysis acid diluted $(\mathrm{HCl})$ with percentage $(40 \%)$, then it is placed in alcohol Pure ethanol (99\%) for (10) $\mathrm{min}$, then in the acetone to remove the residue of the oil impurities if it is good [15] for (15) min. The prepared mixture, deposited homogenously in the middle of the glass bases then put into the spin coating $1500 \mathrm{rpm}$ for 30 seconds, then placed in a heat oven for the process of annealing of (50C0)for 2 hours. to be ready for use, then weight the substrates using a sensitive balance (10-4 mg).Spin coating is currently the technique employed to produce uniform thin films of photosensitive organic materials with thickness of the order of micrometers and nanometers

\section{Results and Discussion}


One of the most important means of determining the bands structure of semiconductors is the optical measurements and light transitions between the energy bands that determine the optical gap; optical permeability of the $\mathrm{ZnTiO} 3$ polymer nanocomposite with $(0 \mathrm{Wt} \%, 1 \mathrm{Wt} \%$, $2 \mathrm{Wt} \%$, $3 \mathrm{Wt} \%$, 4Wt $\%)$ thin films was measured figure (1) shows the transmittance spectrum of these thin films for $(0 \mathrm{Wt} \%, 1 \mathrm{Wt} \%, 2 \mathrm{Wt} \%$, $3 \mathrm{Wt} \%$, 4Wt\%) ZnTiO3 nanocomposite with wave lengths of range (200-1100) $\mathrm{nm}$ is a drop in the ultraviolet spectrum (200-350) and (350-1100) $\mathrm{nm}$ and this indicates the high absorption of the falling photon on the thin film in this area of spectrum, as we note that transmittance of the thin films increases by increasing the wavelength $(300) \mathrm{nm}$ to $(70 \%, 64 \%, 54 \%, 46 \%, 56 \%)$ for $(0 \mathrm{Wt} \%, 1 \mathrm{Wt} \%, 2 \mathrm{Wt} \%, 3 \mathrm{Wt} \%, 4 \mathrm{Wt} \%)$ ZnTiO3polymer nanocomposite respectively gives a good agreement comparing with other ref[6,7], were in the two regions of the visible and infrared spectrum of $(1 \mathrm{Wt} \%)$ we show peak of transmittance while the area (500-800) nm of spectrum increased absorption which indicates shows the appearance of absorption bands for the prepared materials in this region of the spectrum Returns to the transfer of the charge from the valance band to the conductive band, figure(2) shows the absorption coefficient of the system $\mathrm{ZnTiO} 3$ polymer nanocomposite by weight ratio $(0 \mathrm{Wt} \%, 1 \mathrm{Wt} \%, 2 \mathrm{Wt} \%, 3 \mathrm{Wt} \%, 4 \mathrm{Wt} \%)$ respectively, absorption coefficient is dependent on the energy of falling photons and semiconductor properties(energy gap) and the type of electronic transitions that occur between energy bands, that absorption depends on a combination of factors such as: type and nature of the thin film also thickness of the membrane type and proportion of added reinforcement material . From Figure (2) note that the absorption coefficient decreases with increasing photon energy this is due to increased permeability within the wavelength (300-1100)nm this indicates a high probability of electronic transitions The indirect and high energy at which this value is calculated is the energy of a indirect energy gap, also we observe from the figure that the value of the absorption coefficient be few at low photonic energies In which the probability of electronic transitions is low. The absorption coefficient value increases at the edge of absorption towards high energies because at the edge (or basic absorption) The probability of photon absorption is high and therefore increases absorption reduces permeability and increases the absorption coefficient. Figure (3) shows the optical energy gap found in the relationship plot between $(\alpha h v) 2$ and $(h v)$ so from the intersection of the straight part to the curve $(\alpha h v) 2$ equal (zero), the intersection of these straight lines with the coordinates of photon energy determines the value of the optical energy gap to the thin films of ZnTiO3 polymer nanocomposite, table (1) shows the optical energy gap of the $\mathrm{ZnTiO} 3$ polymer nanocomposite thin films which can be determined from the absorption edge because it indicates the electronic transitions from the valance band to the conduction ban. The highest value of the energy gap for the prepared thin films was at pure epoxy resin which equal to(3.4 ev), then $1 \%(3.1 \mathrm{ev})$ and $2 \%(2 \mathrm{ev})$ and $3 \%(3.2 \mathrm{ev})$ attributed to the topical levels between the valance band and the conduction band due to the appearance of absorption bands in the region 200-350 nm of spectrum. In general the reason is due to variation in the amount of the additive and this is due to agglomeration winning in the polymer matrix at $2 \mathrm{wt} \%$ (2ev) (the energy gap is reduced by increasing the ratio of the reinforcement material to the polymer: this leads to new topical levels above the valence band and below the connection band. these levels are ready to receive the electrons and generate tails in the forbidden gap. these tails work towards reducing the energy gap. It is one of the crystal defects. Fourier Transform Infrared Analysis (FT-IR) devise used to identify the bonding between the polymer (matrix) and nanoparticles; Infrared spectroscopy is the study of interactions between matter and electromagnetic fields in the IR region. In general, a frequency will be strongly absorbed if its photon energy coincides with the vibration energy levels of the molecule. Figure (4) shows the system reaction of epoxy resin while Figure (5) shows peak ranges and specific bonds indicated by the peaks previously found of Epoxy, These observations are clear indication that grafts copolymerization has occurred between (matrix and monomer) components. For pure epoxy a strong band at $752.26 \mathrm{~cm}^{-1}$ the spectrum corresponds to the stretching vibration of the $\mathrm{O}-\mathrm{H}$ bond [17] , $\mathrm{C}-\mathrm{H}$ bond is broken, lower absorbance band at rang $2843.17-3441.12$, at (1w\%) 2951.19-2999.41 $\mathrm{cm}^{-1}$ lower absorbance $\mathrm{C}-\mathrm{H}$ bond is broken $1444.73 \mathrm{~cm}^{-1}$ Vibration intensity appear at $2999.41 \mathrm{~cm}^{-1}$ and $2951.19 \mathrm{~cm}^{-1}$, The - $\mathrm{CH} 2$ substituent shows the absorption peaks around $2950 \mathrm{~cm}^{-1}$ due to the $\mathrm{CH}$ stretching band in methyl and methylene groups. This high absorption rate and its continuity even at higher wave lengths are due to the improvement of epoxy resin by (\%additive) of nano particles of $\mathrm{ZnTiO} 3$. 


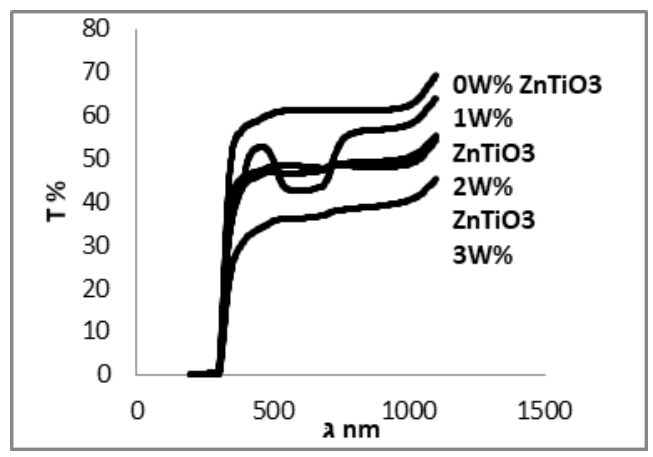

Fig. (1) Transmittance spectrum for ZnTio3-Epoxy nanocomposite thin films with wave length

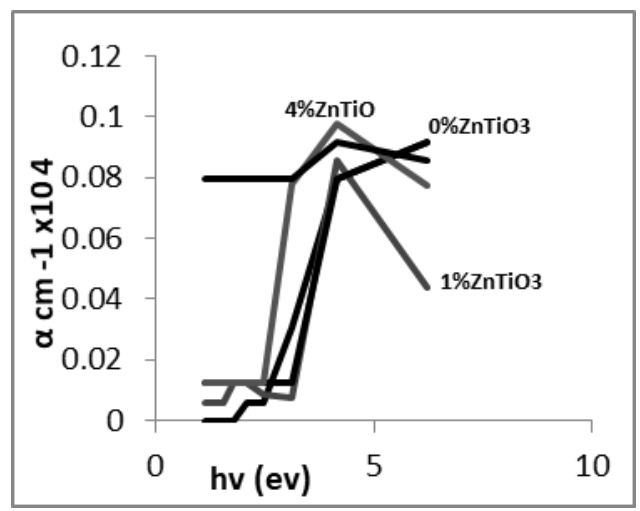

Fig. (2) Absorption coefficient for ZnTio3-Epoxy nanocomposite thin films with photon energy

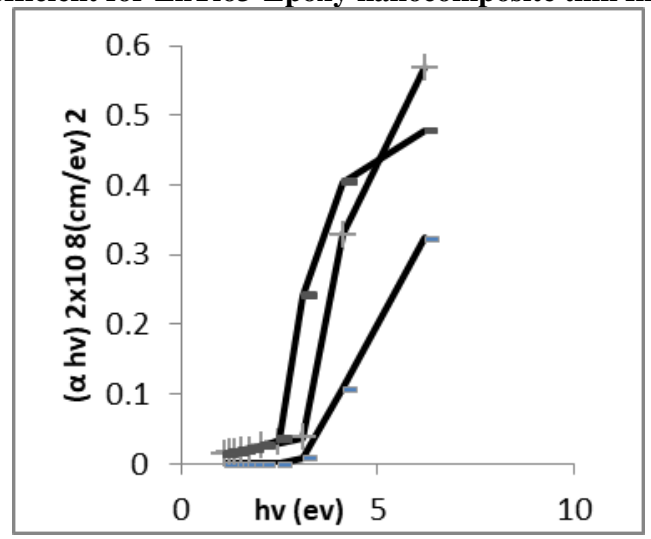

Fig. (3) Optical energy gap for ZnTio3-Epoxy nanocomposite thin films for difference Wt\%

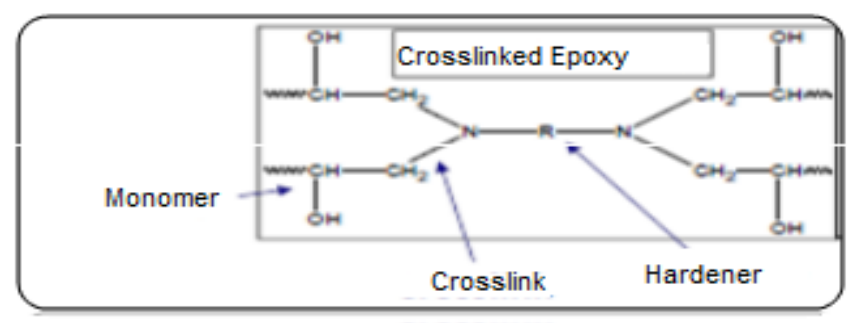

Fig 4. System reaction of epoxy resin

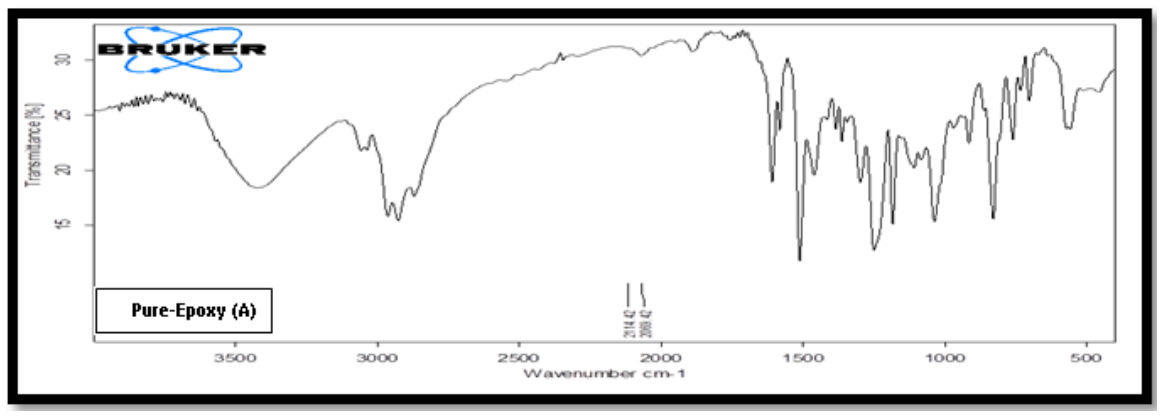

Obongo Matibe Bernard, IJSRM Volume 06 Issue 10 October 2018 [www.ijsrm.in] $\quad$ EM-2018-103 

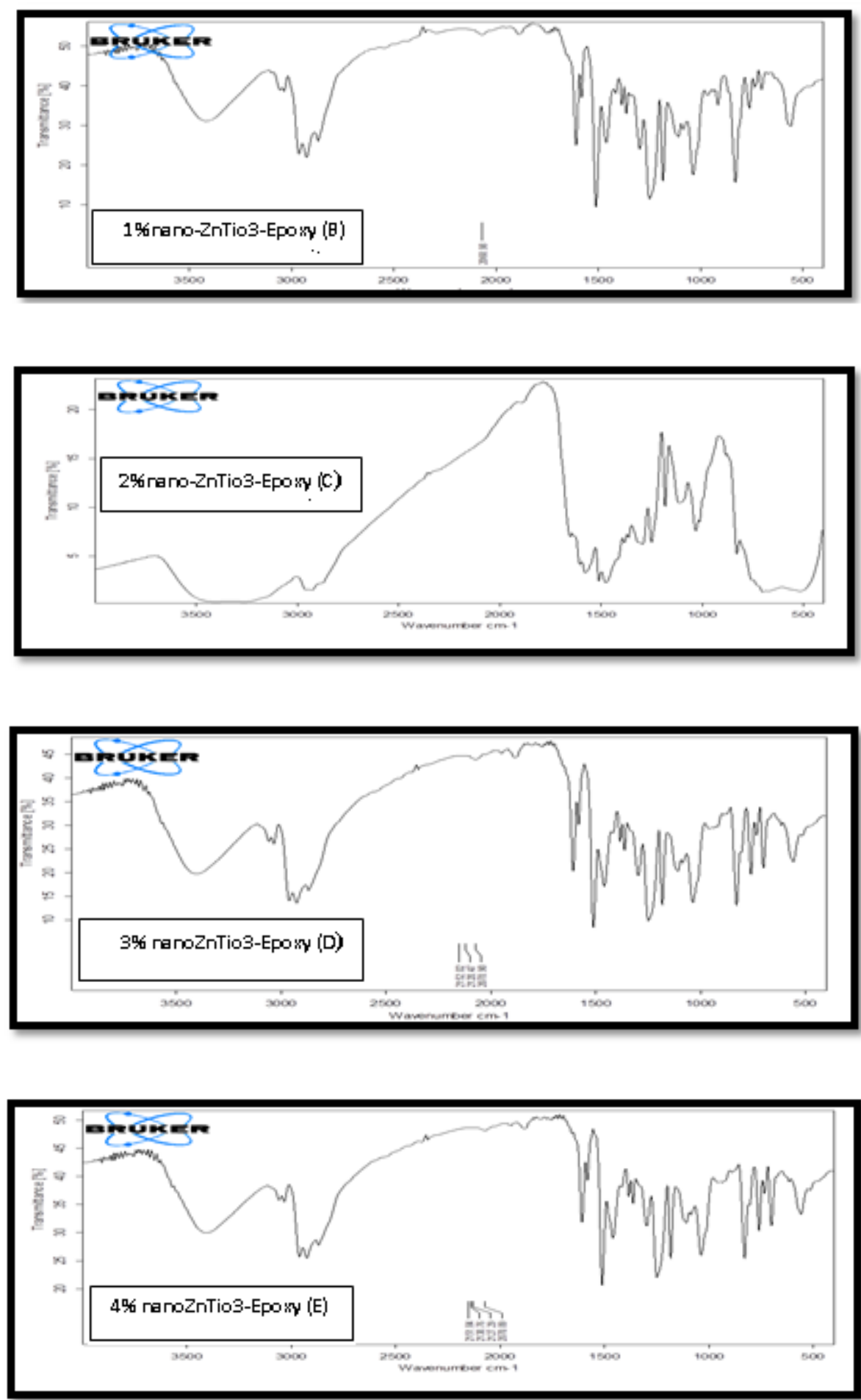

Fig. (5) For ZnTio3-Epoxy nanocomposite thin films (A: 0\%, B: 1\%, C: 2\%, D: 3\%, E4\%) Fig. (5) FT-IR

Table (1) showed values of energy gap for difference $\mathrm{Wt} \%$ 


\begin{tabular}{|c|r|c|}
\hline Weight fraction \% & Transition type & Energy gap (ev) \\
\hline \hline Pure Epoxy & Indirect allowed & 3.4 \\
\hline $1 \%$ ZnT io3-Epoxy & Indirect allowed & 3.1 \\
\hline $2 \%$ ZnTio3-Epoxy & Indirect allowed & 2 \\
\hline $3 \%$ ZnTia3-Epoxy & Indirect allowed & 3.2 \\
\hline $4 \%$ ZnTia3-E poxy & Indirect allowed & 2.5 \\
\hline
\end{tabular}

5- Conclusions

1- For the uv region, we observe a decrease in the permeability spectrum of $\mathrm{ZnTiO} 3 /$ epoxy nanocomposite thin films there is absorption bands in the (UV) regions (200-300) nm at the ratio 0\%wt, (200-350) nm for $(1 \% \mathrm{wt})$, and for $(2 \% \mathrm{wt}, 3 \% \mathrm{wt}, 4 \% \mathrm{wt})$ also there is absorption bans only in the (UV) region and have

2-Note the low absorption coefficient of $0 \mathrm{wt} \%$ then increase by increasing the weight ratios with increasing wavelength

3- Energy gap for ZnTiO3/ epoxy nanocomposite suitable for solar cell fields

\section{References}

[1] C. A. Harper, Electronic Packaging and Interconnection Handbook, McGraw Hill (2004)

[2] Chanda M., Introduction to Polymer Science and Chemistry, CRC Press, Taylor and Francis Group, FL, USA, 2006.

[3] Caseri W. Inorganic nanoparticles as optically effective additives for polymers. Chem Eng Comm. 196: 549-72. [Taylor \& Francis Online] [Google Scholar] (2009).

[4] A. Endruweit, F. Gommer, A.C. Long. Stochastic analysis of fibre volume fraction and Permeability in fibre bundles with random filament arrangement, Composites Part A: Applied Science and Manufacturing, Volume 49, Pages 109-118, ISSN 1359- 835X, June (2013).

[5] S. H. Wei and A. Zunger, Appl. Phys. Lett. 72, (2011).

[6] H.Bensmira, Thése de magister. Université Mentouri, Constantine (2004) .

[7] U. diebdd. Surface Science reports. 48, 53-229 . (2003)

[8] Jarmila M"ullerov’a (Optical properties of zinc titanate ZnTiO3prepared by reactive RF PutteringJournal of ELECTRICAL ENGINEERING), VOL 68, NO7, P 10-16, (2017

[9] H. Hafez, Z. Lan and Q. Li, Highly Efficient Dye-sensitized Solar Cells Based on Single Crystalline TiO2 Nanorod Film, Chem. Lett., 34, 1506-1507 (2005)

[10] Al-Jubory A. ,Alaa"Study the structural and Optical properties of Zni-xPbxS thin Films which preparing by Chemical spray Pyrolysis", N.Sc.Thesis, Gollage of Since , Al-Anbar University, p (44),(2005)"

[11] Kasap S.O.,"Principles of Electronic Materials and Devices", Snd Ed, Mc Graw-Hill, New York, (2002).

[12] EL-Mallawany.R, Abadlla .M.D ๑®Abbas.A.I, , Materials Chemistry @Physics, 109, pp. 291296(2008).

[13] R.K.Fakher Alfahed, K.I.Ajeel, International Journal of Technology Enhancement and Emerging Engineering Research, Issue 08 ,3, Issn 2347-4289 pp.36-41,( 2015

[14] J. S. Blakmore, "Solid State Physics", Gambridge Press . 11 p1810-1814(2009)

[15] Xia H.S., Wang Q. Preparation of conductive polyaniline/nanosilica particle Composites through ultrasonic irradiation. J. Appl. Polym. Sci; 87:1811-1817. Doi: 10.1002/app.11627. ( 2003) 
[16] Joshi M., Khanna R., Shekhar R., Jha K. Chitosan nanocoating on cotton textile Substrate Using layer-by-layer self-assembly technique. J. Appl. Polym. $\quad$ Sci. 119:2793- 2799. Doi: 10.1002/app.32867 ( 2011).

[17] Mustafa Oztas, Metin Bedir, " Thickness dependence of Structural, electrical and Optical properties of sprayed ZnO: Cu films " Thin Solid Films Vol. 516 P.1703-1709, (2008). 